\title{
Triangulation Measurements in the Solar System
}

\author{
V.K. Abalakin, M.S. Chubey, G.I. Eroshkin, I.M. Kopylov \\ Pulkovo Astronomical Observatory 65/1 Pulkovskoye shosse, \\ Saint-Petersburg, 196140, Russia E-mail: vicabal@gao.spb.ru; \\ mchubey@gao.spb.ru
}

\begin{abstract}
The operational work of the Interplanetary Solar Stereoscopic Observatory (ISSO) may continue for 11 years. The ISSO Project is designed to insert two spacecraft (SC) into Lagrangian triangular libration points of the "Sun + barycenter (the Earth + the Moon)" system. The Project scientific program consists of two sets of experiments related to solar physics and to dynamical astronomy, respectively.

Various options of the astronomical observation program are possible. We discuss the option where the stereoscopic mode is applied to the direct triangulation observations of the solar system objects with respect to the ICRS coordinate frame.

The stereoscopic mode with the baseline equal to $\sqrt{3}$ appears to be suitable for receiving the three-dimensional (3-D) measurement series for planets during the ISSO's lifetime. Extended investigation of the planetary aberration based on the observations of major and minor planets, the direct distance determinations for minor planets and Kuiper's belt objects, and the new set of star parallaxes may be used for constructing a new fundamental ephemeris and establishing an alternative scale of stellar distances. The accuracy of the angular measurements is expected to attain the milliarcsecond level.
\end{abstract}

\section{Introduction}

Stereoscopic vision with the base-line of $\sim 6-7 \mathrm{~cm}$ had been the first natural basis of human evolution in its geometric 3-D adaptation in the world. It is just that principle that is laid in origin of all scale systems in modern astronomy as well. The maximum usage of the principle is possible in the stable stereoscopic mode of astronomical observations.

The Project of the Interplanetary Solar Stereoscopic Observatory (ISSO) has been proposed by Grigoryev (1993). Extended scientific objectives and programs were proposed by Chubey et al., (1998). Besides the 3-D monitoring of solar activity processes, the essential advantages for observations of practically all solar system objects and of star parallaxes in the mode of direct triangulation are evident.

The observations of the microvariable stars, macro- and microlensed objects can be also performed by the ISSO instruments. But neither this topic, nor the solar monitoring program will be described in this article as being out of the 
main scope of this IAU Colloquium. We shall not be concerned also with detailed aspects of the Project except the observation instruments.

Our main goal is to describe, strictly speaking, the triangulation potentialities of the ISSO.

Two identical on-board instrumental complexes are supposed to be used to solve the astronomical problems in the stereoscope mode and/or in the separate programs for the direct imaging of the celestial bodies with differential astrometric determinations of the object positions in the reference catalog system and to determine the images' brightness using the broadband photometry system UBVR. The standard calibration of the instruments and the accurate chronometry of all measuring processes will be performed under the constant control of an on-board computer.

\section{The optimal launching scheme}

Inserting the spacecraft (SC) into the libration points $L_{4}$ and $L_{5}$ (Fig. 1) can be made in the framework of the three-pulse orbit transfer scheme. Thus the start of the SC, aiming at insertion into the point $L_{5}$, occurs from a near-Earth orbit first at time $D_{1}$ when the Earth is at the point $L_{5}$. For the transition orbit the given point coincides with the perihelion. After 1.17 years when this $\mathrm{SC}$ will return again to its starting point, it will brake and stop at this point of the Earth orbit, which will be the Lagrangian point $L_{5}$. In the time span of the motion of this SC the Earth will have moved around the Sun by the angle of $420^{\circ}$ and will be advancing the SC in the orbit by the angle of $60^{\circ}$.

At the time moment $D_{2}=D_{1}+0.34$ years, when the Earth will be at the point $L_{4}$, the start from the near Earth orbit of the second SC in the framework of the same scheme should be made, but for this transition orbit the starting point will be now at the aphelion. This SC will return to the same point in 0.83 years. At time $D_{3}=D_{1}+1.17$ years, both spacecraft simultaneously arrive at the Lagrangian points and their decelerating (braking) or accelerating, and the maneuvers to form final orbits also will be made. The Earth at the moment $D_{3}$ occupies its pre-calculated position $T_{3}$, and the stage of the $\mathrm{SC}$ navigation will be completed. This sequence of inserting the SC into their orbits is the optimal one for decreasing the time of radiation influence upon the yet undeployed scientific equipment (Chuchkov, 1989; Zabelina, 1997).

The spacecraft with instruments are placed in Earth orbit in the points $L_{4}$, $L_{5}$ (Fig. 1) which are the vertices of the equilateral triangles $S L_{4} T$ and $S L_{5} T$ where $T$ means the Earth's position in its orbit.

To strictly meet the metrology requirements, in addition to the calibration measurements of distances by a radar system, it is necessary to determine the angles between the sides $L_{4} T_{3}$ and $L_{5} T_{3}$ of the triangle (Fig. 1). A method of measurement of the angles as shown in Fig. 2 is based on synchronous measurements of the positions of the Earth and the Moon images with respect to the stellar frame of reference represented by a reference catalog.

The same observation mode for major planets or asteroids. with well-developed theories of motion is assumed to be used for the regular determination of the distances $L_{4} T_{3}$ and $L_{5} T_{3}$. The DE403/LE403 ephemerides are to be used as the references for this navigation as well as other program requirements. 


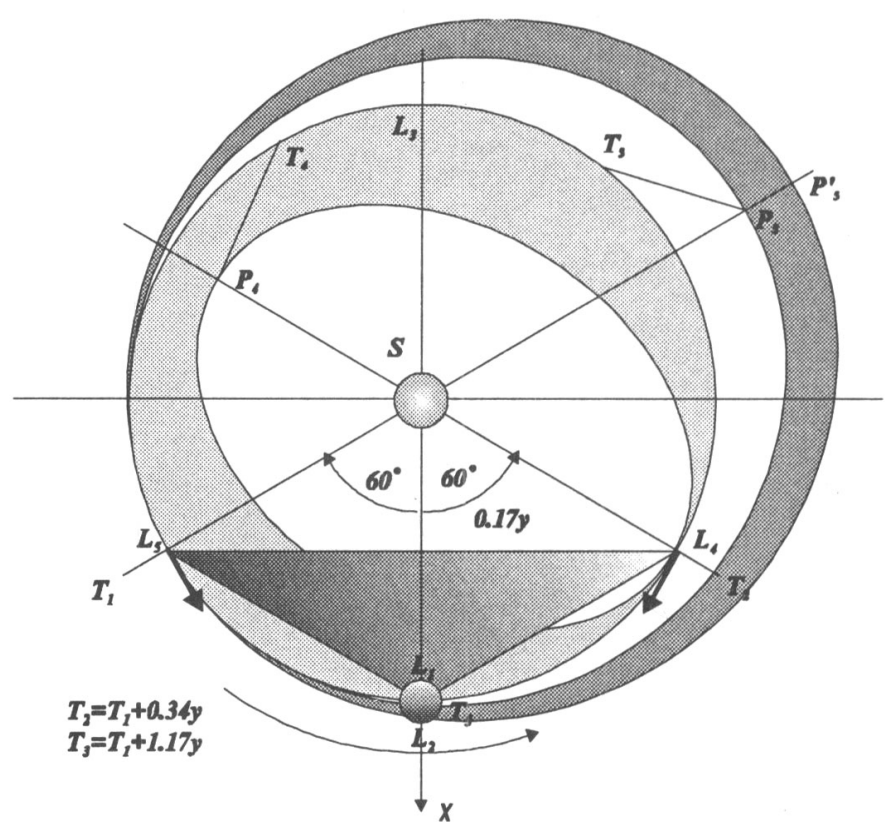

Figure 1. Optimum scheme of SC starts and the formation of their orbits. $P_{5}$, the aphelion of the transporting orbit for the spacecraft in $L_{5}$, the Earth being at $T_{5}$ at the moment of the aphelion $P_{5}$ passage. $P_{4}$ is the analogous aphelion for the spacecraft placed in $L_{4}$. The small deviation in the SC start velocity may result in the essential change of the heliocentric transfer path and time, so that permanent orbital control is necessary. 

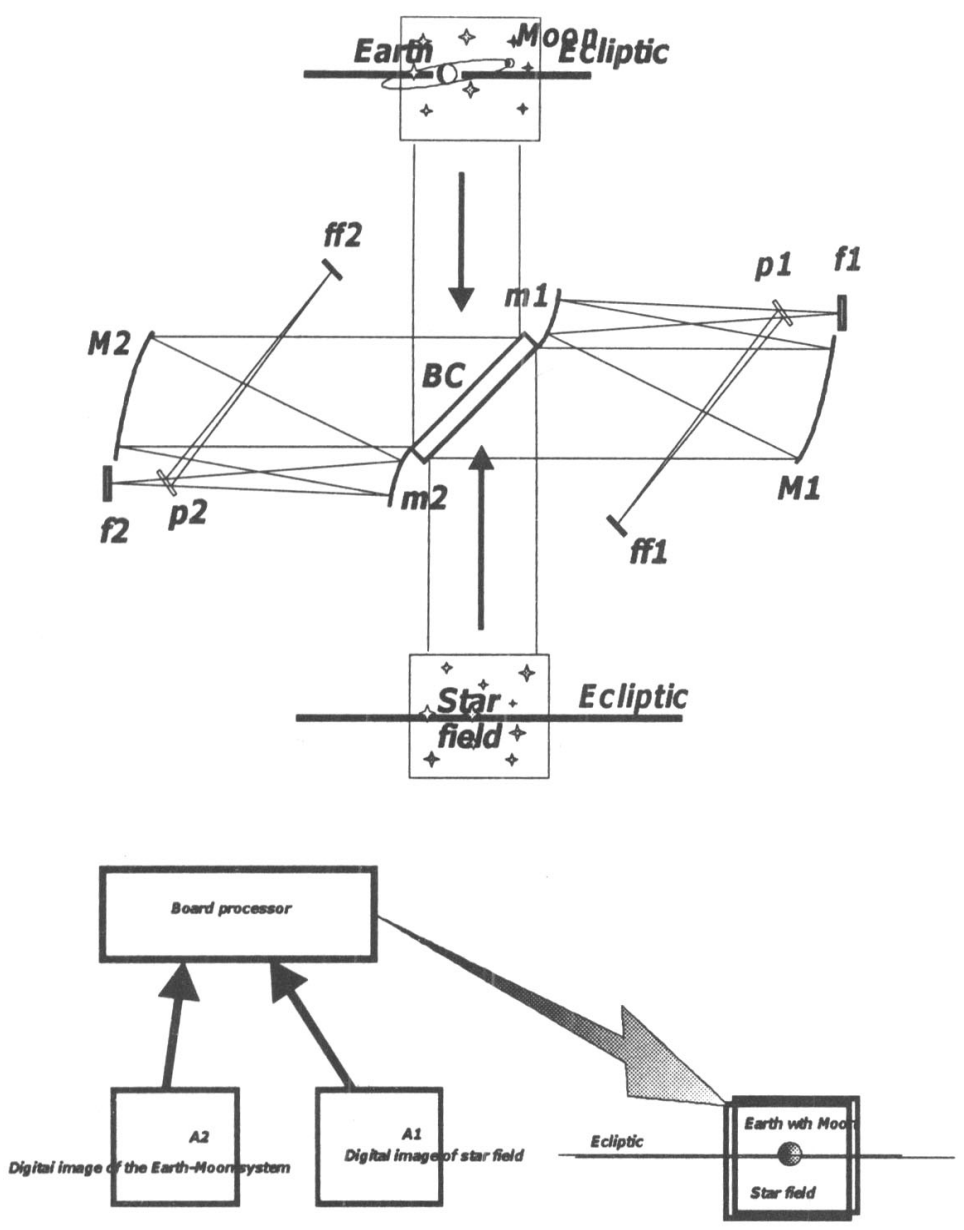

Figure 2. The remote self-navigation instrument concept. The bright $\ll$ Earth + Moon $\gg$ will be imaged in the focal planes $f 2$ or $f f 2$. The image of the undisturbed $\ll$ starfield $\gg$ which is placed on the extension of the vector $T L_{5}$ (or $T L_{4}$ ) will be imaged in the planes $f 1$ or $f f 1$. The on-board processor analysis gives the vector orientation. 
The Earth's image as seen from the points $L_{4}$ and $L_{5}$ will look like a sphere (more precisely, like an ellipsoid of rotation) not completely illuminated by the Sun with the angular diameter $d e=17 " 6$, the constant phase angle $\alpha=60^{\circ}$, and with the position angle of the dark terminator $\chi=0^{\circ}$. The integral brightness of the Earth and the Moon in the V-filter will be $-2.95^{m}$ and $+1.84^{m}$, respectively. Their observations as well as the observations of bright stars and planets require absorption filters to be used, and a special processing technique as well.

The possibility has not been excluded that the same observations of the Earth will possibly provide for highly important photometry information about the global processes in the terrestrial ionosphere and magnetosphere that may be detected only from remote long-term observations.

\section{Dynamical aspects of the experiment}

The numerical simulation of the inertial motion of the $\mathrm{SC}$ is performed by the simultaneous integration of the post-Newtonian orbital motion equations for the Sun, major planets, the Earth + Moon barycenter, the Moon, the two zero masspoints (the SC) as well as of the Newtonian equations of the Moon's rotation. The initial conditions of the SC motion are defined in the first approximation by the position, velocity and acceleration of the Earth + Moon barycenter and the coordinates of the triangular libration points $L_{4}$ and $L_{5}$. The integrated equations of motion of the major bodies of the solar system reproduce rather well the fundamental ephemeris DE200/LE200. Thus the predicted orbits of the $\mathrm{SC}$ can be regarded as authentic.

If the initial conditions are formed with no deviations from the theoretical ones, then the motions of SC in the vicinity of the $L_{4}$ and $L_{5}$ points would be rather regular ones.

The orbital motion of the zero-mass bodies in the vicinity of the Lagrangian libration centers $L_{4}$ and $L_{5}$ is shown in Fig. 3 in terms of variations of the angles $L_{5} S T_{3}, L_{4} S T_{3}$ (Fig. 1). It is necessary to emphasize that the algorithm to construct the SC's orbits is completely identical to the one used to construct the major planets' orbits. It is necessary to complement the system of the differential equations of the problem under consideration by the differential equations of the Earth's rotation. It permits us to predict with required precision the positions of the SC with respect to an arbitrary point on the Earth's surface. It is quite necessary for practical computation of the initial conditions of passive motion, as well as for the guidance and the full-scale metrology control.

Synchronous observations permit us to exclude both the motion of an object and that of an observer. The series of star observations is subject to errors of different origin and properties in comparison with classical ones.

\section{Construction of the spacecraft orbits. Determination of the Stere- oscope base-line}

The idea of the method is based on application of the on-board, two-directions star sensor (Fig. 2) allowing measurements in a coordinate system of a highprecision. It is assumed that for a known moment of observation the available on-board equipment includes: 


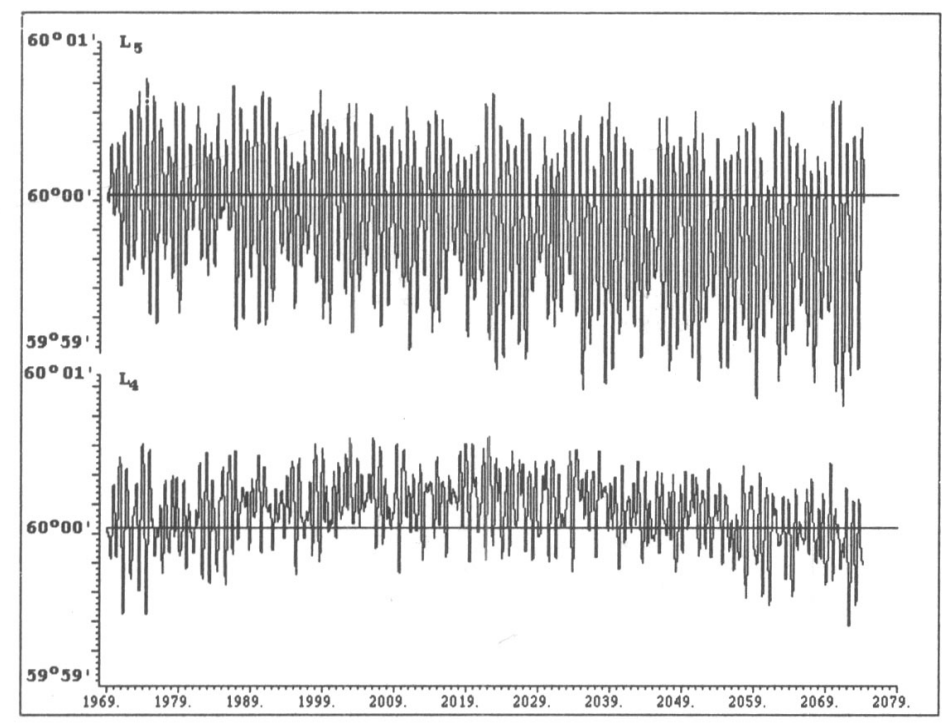

Figure 3. The post-Newtonian approximation of the motion of zero mass-points in the vicinity of the triangular libration points over the time span from 1969 to 2075 as represented by variations of the heliocentric angles between the Earth + Moon barycenter direction and the zero mass-points directions.

- The software package for computing the position ephemerides for the Earth $(T$, Fig. 1), the Moon (Moon), observed planets $(P l)$ and their satellites $(S a t)$, and minor planets $(M p l)$;

- Catalog and software for an evaluation of astrographic positions and brightness of stellar objects in required areas on the sky;

- The numerical theory of motion of zero mass-points (used for the SC, each $\mathrm{SC}$ is treated as a third body in the three-body problem) in the vicinity of the libration centers;

- The on-board processor with an appropriate operating system and a clock (the time standard).

By using the star sensor the following problems of spatial position determination of a spacecraft have been solved for two cases:

1. The spacecraft position determination from the on-board angular measurements only,

2. The spacecraft position determination when the direct radio measurements of distances from a ground-based command-measuring complex to the spacecraft are available.

Let's consider each case separately. 


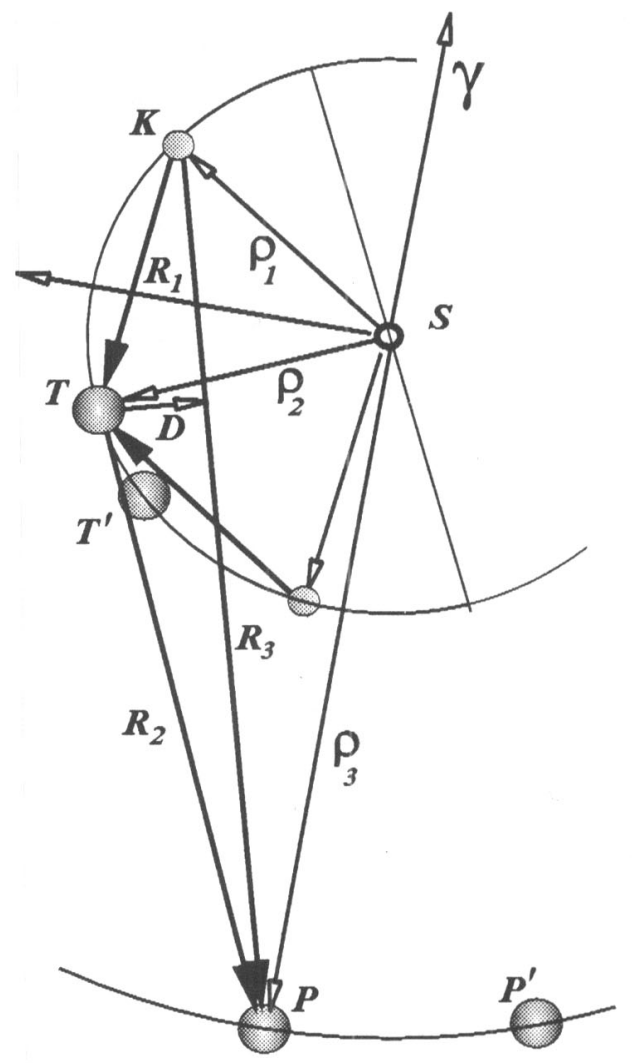

Figure 4. Determination of the proper position of a spacecraft by observing the Earth and a planet or the Earth and the Sun.

\subsection{The spacecraft position determination from the on-board angu- lar measurements only}

Such a determination may be executed at all phases of the ISSO lifetime, i.e. from the moment of transition onto a heliocentric transfer orbit to the complete conclusion of the experiment. Directions of two vectors $R_{1}$ and $R_{3}$ (Fig. 4) are independently being measured by the star sensor on-board the SC. The times of events are recorded by the on-board clock and compared with the scale of Barycentric Dynamic Time, TDB. The vector $R_{2}$, from the Earth's center to the planet's center is calculated on-board using a standard ephemeris giving the Earth's and a planet's heliocentric radii vectors $\rho_{2}$ and $\rho_{3}$, respectively, at the required moment. Hence, in the triangle $K T P$ the linear element, the modulus of the vector $R_{2}$ is calculated, and the directions of vectors $R_{1}, R_{3}$ and $R_{2}$ are measured. The determination of the modulus of vector $R_{1}$ now becomes possible because the linear element and all three angles in the triangle KTP are known. 
Actually, through three points only one plane can pass, and, therefore, the vectors are coplanar. The vector $R_{2}=T P=\left\{r_{2}, \lambda_{2}, \beta_{2}\right\}$ direction is measured from a point $K$ in the spherical geocentric coordinate system. The vector is calculated using the Earth $T$ and a "navigational" planet $P$ radius vectors $\rho_{2}$ and $\rho_{3}$, respectively. The direction of vector $R_{3}=T P=\left\{r_{3}, \lambda_{3}, \beta_{3}\right\}$ is also observed from the point $K$. Taking into account, if necessary, the parallactic displacement of reference stars in the frame as recorded by a navigational observation of the planet $P$ and caused by the shift along the vector $D$ directed perpendicularly from the Earth's center onto the vector $R_{3}$ direction (Fig. 4) we can reduce the vector $R_{3}$ to the Earth's center. Now in the flat triangle $K T P$ the angles at its vertices are those formed by the known directions, i.e. they also are known. So, the triangle may be solved, and the vectors $R_{1}, R_{3}$ determined.

All three bodies are moving, but it is easy to consider their motions during the observation time span as circular (probably, a linear approximation will be sufficient). To measure all directions and line segments entering the navigation and actually belonging to the effective unified triangle in Euclidean space, it is necessary to take into account changes of spatial positions of three bodies, the centers of which form the navigational triangle during the appropriate light-time intervals. To determine the values of these light-time intervals and to correctly calculate the ephemeris positions of the Earth and the planet for an observation, let us consider again Fig. 4.

Let's assume that the observations of directions of vectors $R_{1}, R_{3}$ are made at a point $K$ at the common time $t_{\text {obs }}$. It is possible to optimize the sequence of observations. The measured angle $\angle T K P=\phi_{1}$ is the angular distance between two points in the satellite-centric coordinate system $K(r, \lambda, \beta)$, the axes of which are parallel to those of the heliocentric ecliptic coordinate system. The first point $T\left\{r_{1}, \lambda_{1}, \beta_{1}\right\}$ coincides with the heliocentric position $\rho_{2}$ of the Earth $T$ at the moment preceding the moment $t_{o b s}$ by the time-span required for the light wave to travel over the distance $r_{1}$. The second point $\left\{r_{3}, \lambda_{3}, \beta_{3}\right\}$ represents the analogous position $\rho_{3}$ of a planet $P$ at the moment preceding the moment $t_{o b s}$ by the value of the corresponding light-time interval. Let's designate $\angle T P K=\phi_{3}$ and $\angle K T P=\phi_{2}$.

The light-time $\tau\left(R_{i}\right)$ for the vector $R_{i}$ is calculated (but not measured!) by the formula:

$$
\tau\left(\vec{R}_{i}\right)=\frac{\left|\vec{R}_{i}\right|}{c}
$$

if both the modulus of the vector and the velocity of light in vacuo are known. To determine the vector $R_{3}$ now we have the vector equation:

$$
\overrightarrow{R_{2}}\left(t_{o b s}\right)=\overrightarrow{\rho_{3}}\left(t_{o b s}-\frac{\left|\overrightarrow{R_{3}}\right|}{c}\right)-\overrightarrow{\rho_{2}}\left(t_{o b s}-\frac{\left|\overrightarrow{R_{3}}\right|}{c}\right) .
$$

The sine formula gives the following values of the vectors $R_{1}, R_{3}$, their directions in space having immediately been observed:

$$
\left|\vec{R}_{1}\right|=\left|\vec{R}_{2}\right| \cdot \frac{\sin \phi_{2}}{\sin \phi_{1}}
$$




$$
\left|\vec{R}_{3}\right|=\left|\vec{R}_{2}\right| \cdot \frac{\sin \phi_{2}}{\sin \phi_{2}} .
$$

All variables entering the formulae (2), (3) are functions of the light-time. For the evaluation of the light-time the equations (1), (2), (3) should be solved by iteration in accordance with the scheme:

$$
\begin{array}{r}
\tau_{0}=0, \rightarrow \vec{R}_{k}=\vec{R}_{k}\left(\tau_{k-1}\right), \\
\tau_{k}=\frac{\left|\vec{R}_{k}\right|}{c}, k=1,2 \ldots \\
\left|\tau_{k+1}-\tau_{k}\right| \leq \varepsilon,
\end{array}
$$

where $\varepsilon$ is the required accuracy of an evaluation of the time argument.

\subsection{Determination of the spacecraft position using the direct ra- dio measurements of distances from a ground-based command- measuring complex (GCMC) to a spacecraft. Evaluation of the stereoscope base-line.}

Let's assume that the deep space communication radar combined with the retranslator located onboard a spacecraft are used to measure distances from GCMC to both spacecraft. In this case the measurement scheme provides the absolute (not the pre-computed) range tracings.

The radar measurements give the UTC proper time intervals of the radio waves propagation by use of the atomic clock at an observing site. Let's consider the true path of a radio beam.

It is impossible to observe directly the position of the "Earth + Moon" system barycenter. The Earth is observed, and its position with respect to the " $E+M$ " barycenter is known. The time record $t$ is made on the scale of ephemèris time TDB (the Barycentric Dynamical Time), being kept, e.g., by the atomic time standard of an observing site.

In an ideal case the vector of the base-line $K P$ (Fig. 5) connects the centers of spacecraft $K$ and $P$ in their theoretical position relative to the barycenter of the system « "the barycenter of the Earth + the Moon" system + the Sun».

Due to the influence of the planetary aberration and of the pure libration motion the observables are the vectors $R_{1}, R_{2}$, and the angle $K^{\prime} T P^{\prime}$. At a moment $t_{0}$, let a signal be emitted from the Earth as the spherical wave $v$ propagating with the velocity of light. Because of orbital motions of the centers $T, K$ and $P$ the instrument on the SC moving in the vicinity of the libration center $L_{5}$ will meet the wave $v$ at the point $K$ at the time $t_{K^{\prime}}=t_{0}+\tau\left(R_{1}\right)$, while that aboard the SC moving in the vicinity of the $L_{4}$ center meets the wave at the point $P^{\prime}$ at the time $t_{P^{\prime}}=t_{0}+\tau\left(R_{2}\right)$. At the moments of their arrival at the SCs, let the signals be reflected in the direction to the pre-computed Earth position at the moments of arrival of the reflected signals from both spacecraft. Obviously, it is the position of the Earth at $T^{\prime}$ where the reflected signals from both SCs will meet since the path of the ray in the direction of the orbital motion of the Earth and in the opposite direction will be identical, so that $\left|\vec{R}_{2}\right|=\left|\vec{R}_{4}\right|$. Similarly, one gets $\left|\vec{R}_{1}\right|=\left|\vec{R}_{5}\right|$. It should be noted that the rotational shift of 


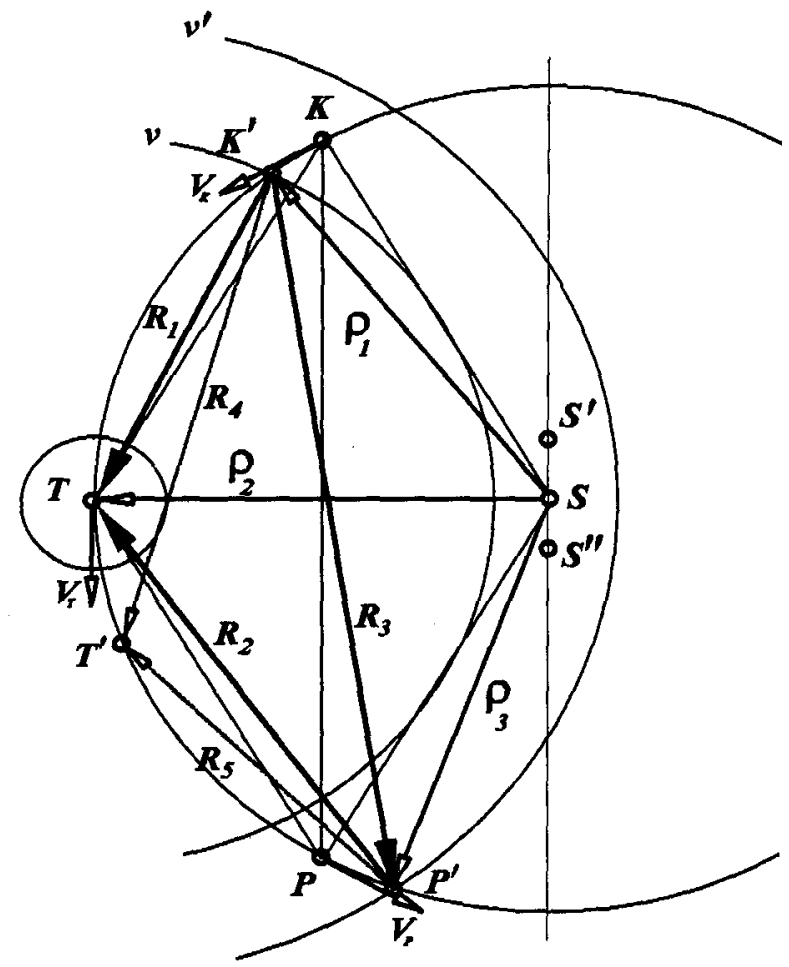

Figure 5. The concept of triangulation measurements of the Stereoscope baseline vector. $S^{\prime} S^{\prime \prime}$ is the line of apses of the Earth orbit. 


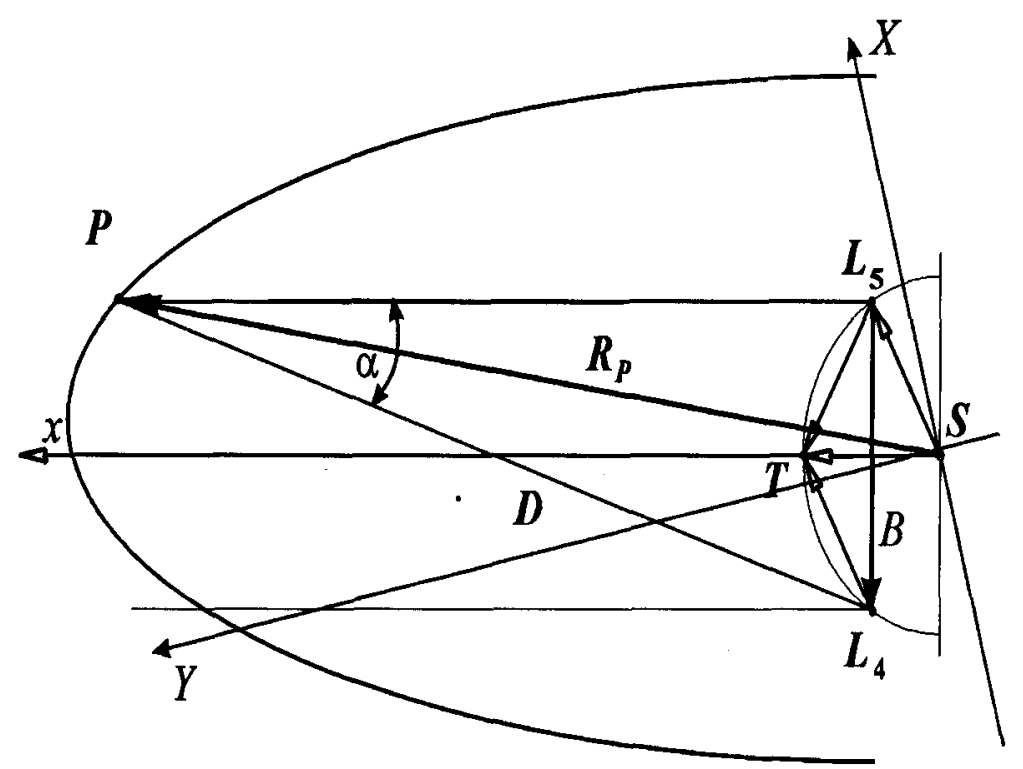

Figure 6. The elementary scheme of measurements of a radius vector for a body of the solar system.

the observing site, the delay in the Earth's ionosphere, and the correction terms of the second order in evaluating the light-time intervals have been neglected here (Standish, 1990). We note only that in our case the radio signals are not reflected from the surface of a planet which has its own atmosphere and radiation belts but from points of the antennas aboard the spacecraft.

Thus, the navigational triangles are formed by the point $T$ from which the signal is emitted at the time moment $t_{0}$, and by the points $K^{\prime}, P^{\prime}$ and $T^{\prime}$ in which the signal is reflected and received as the echo. To measure the angle $\angle T$ in this triangle, it is necessary to execute the exposures for determination of the Earth's direction at different time moments: $t_{K^{\prime}}=t_{0}+\tau\left(\vec{R}_{1}\right)$ and $t_{P^{\prime}}=t_{0}+\tau\left(\vec{R}_{2}\right)$

Let's measure now the position of the planet $P$ synchronously. In the triangle $K T P$ (Fig. 4) its three angles and the side $L_{4} L_{5}$ are known. The reduction to the heliocentric coordinate system and the solution of a redundant set of equations do not cause any difficulties.

\section{Assessment of the 3-D measurement accuracy}

Let's evaluate the accuracy of the determination of the heliocentric position of a planet from the measurement session.

Let's note that the solution of the problem dealing with the estimation of three-dimensional coordinates of a point object from space stereoscopic observations is already presented in the general review (Bakut and Schulz, 1998) of measurements in near-Earth space. In their measuring system of a stereoscopic pair the absolute observations in the instrumental system were used. Our approach differs in that it is based on differential measurements with respect to the reference frame represented by a high-precision catalog. 
Let the sides $L_{4} T$ and $L_{5} T$ (Fig. 6) be measured in the single session with the error $\sigma_{r}= \pm 20 \mathrm{~km}$. Let's present the vectors $L_{4} T$ and $L_{5} T$ in the spherical heliocentric coordinate system as $L_{4} T=R_{4}\left\{\rho_{4}, \lambda_{4}, \beta_{4}\right\}, L_{5} T=R_{5}\left\{\rho_{5}, \lambda_{5}, \beta_{5}\right\}$. The angle $\gamma$ between the vectors $R_{4}$ and $R_{5}$ we shall find from the formula

$$
\cos \gamma=\sin \beta_{4} \sin \beta_{5}+\cos \beta_{4} \cos \beta_{5} \cos \left(\lambda_{4}-\lambda_{5}\right) .
$$

All arguments in the right-hand side of (5) are measurable quantities and subject to the errors mentioned above. The points $L_{4}, T, L_{5}$ should be located in the plane of ecliptic. After some simplifications we have a differential relationship to determine the error of the computed angle $\gamma$ with respect to the second order terms:

$$
\sin \gamma \cdot d \gamma=\left(\left(\beta_{4}+\beta_{5}\right) \cos \left(\lambda_{4}-\lambda_{5}\right)+\sqrt{2} \sin \left(\lambda_{4}-\lambda_{5}\right)\right) \cdot d \beta .
$$

We can assume that the standard error of a single measurement of the angular component of the direction vector when using the CCD-array observations is $\sigma_{0}=\sigma_{\lambda}=\sigma_{\beta}= \pm 0$ " 05 . Omitting the elementary calculations and taking into account the size of the quantities $\beta_{4}, \beta_{5}$ and the properties of the triangle $L_{4} T L_{5}$, we get the final evaluation of the accuracy of the determination of the angle $\gamma$ as:

$$
\sigma_{\gamma}=\sqrt{2} \sigma_{0}= \pm 0.07=3.5 \cdot 10^{-7} \mathrm{rad} .
$$

Let's find the estimation of the accuracy of the determination of the length of vector $L_{4} L_{5}$ designated as the stereoscope baseline vector $B\left\{\rho_{3}, \lambda_{3}, \beta_{3}\right\}$.

We have the expression for the square of the side in the triangle $\Delta L_{4} T L_{5}$ :

$$
\rho_{3}^{2}=\rho_{4}^{2}+\rho_{5}^{2}-2 \rho_{4} \rho_{5} \cos \gamma .
$$
(8):

We have the complete differentials in the right-hand and left-hand sides of

$$
\rho_{3} d \rho_{3}=\left(\rho_{4}+\rho_{5}\right)(1-\cos \gamma) d \rho+\rho_{4} \rho_{5} \sin \gamma \cdot d \gamma
$$

We shall derive the equation for the dispersion of the measured quantities by squaring both sides of (9) and taking into account that the equalities

$$
\begin{gathered}
\rho_{3}=\sqrt{3} a, \quad d \rho=d \rho_{4}=d \rho_{5}=\left|\sigma_{r}\right|, \quad \cos \gamma=-0.5, \sin \gamma=\sqrt{3} / 2: \\
\left(d \rho_{3}\right)^{2}=\frac{3}{4}(d \rho)^{2}+\frac{a^{2}}{4}(d \gamma)^{2}+\frac{a \sqrt{3}}{2} d \rho \cdot d \gamma .
\end{gathered}
$$

are valid as a first approximation for moduli of the vectors.

By substituting dispersions $d \rho, d \gamma$, we shall obtain the required evaluation of accuracy of the baseline $B$ determination in terms of astronomical units as:

$$
\sigma_{B}=d \rho_{3}= \pm 2.87 \times 10^{-7} \text { a.e. }
$$

Let's evaluate now with what accuracy the distances to planets will be determined by using the stereoscopic baseline $B$. We come back to the scheme of Fig. 6. Let's assume that the planet $P$ is stereoscopically observed in such a 
way that the perpendicular from its center to the baseline vector falls precisely on the extremity of the baseline, i.e. on the point $L_{5}$. We have the rectangular triangle $P L_{5} L_{4}$ in which the side $B$ is known and the angle $\angle P$ is measured, additionally the angle $\angle L_{5}$ is a right angle.

We have the elementary formula connecting the sides and an angle in this triangle:

$$
\sin \alpha=\frac{B}{D}
$$

where $D$ is the perpendicular length or the distance of the planet from the point $L_{5}$.

From (12) we get

$$
D=\frac{B}{\sin \alpha}=\frac{\sqrt{3} a}{\sin \alpha} .
$$

The dispersion of distance $\sigma_{D}$ as that of a function of two variables $B$ and $\alpha$ will be found from the expression

$$
\sigma_{D}= \pm \sqrt{\frac{1}{\sin ^{2} \alpha} \sigma_{B}^{2}+\frac{B^{2}}{t g^{2} \alpha \sin ^{2} \alpha} \sigma_{\alpha}^{2}}
$$

Let's write the complete differentials of the right-hand and left-hand members of the equation (12)

$$
\cos \alpha \cdot d \alpha=\frac{D \cdot d B-B \cdot d D}{D^{2}} .
$$

Thus we shall get the equation

$$
d D=\frac{D}{B} \cdot d B-\frac{D^{2}}{B} \cos \alpha \cdot d \alpha,
$$

which can be written as the equivalent dispersion variations of the measured quantities:

$$
(\delta D)^{2}=\left(\frac{D}{B}\right)^{2} \cdot(\delta B)^{2}+\left(\frac{D^{2}}{B} \cos \alpha\right)^{2} \cdot(\delta \alpha)^{2} .
$$

The contribution to the error of distance determination, made by the baseline determination error, is proporti nnal to the first power of distance $D$. The contribution to this error made by the uncertainty of the angular measurements is proportional to the second power of the distance determined. It follows that the increase of accuracy of the angular measurements is significant to improve the accuracy of triangulation measurements of positions of the solar system bodies.

Another, obvious consequence is that the error of the distance determination decreases with increase of the baseline. In technological and theoretical respects the construction of the baseline using the triangular Lagrange libration points in the system "the barycenter of the Earth + Moon - the Sun" is scientifically justified to the highest degree. 
Results of long-term monitoring of both SC's positions will be the improvement of the Earth's orbit parameters, the determination of the velocity of light in vacuo and the determination of the parameters of the orbital motion of zero mass-points in the vicinity of the libration centers.

With respect to the solar system bodies the most interesting are the triangulation observations of faint objects located at distances $\rho>30$ a.u., i.e. the objects of the Kuiper Belt. Even a single synchronous observation of such an object from the spacecraft allows us to determine all three components of the state vector of an object which would be impossible to find by other methods. Another observation one year later enables us to determine the velocity vector of that far object of the solar system. Derivation of its preliminary elliptical orbit is possible as the solution of the classical Cauchy problem with the initial conditions determined for the moment of the first observation.

It is possible also to make synchronous observations of some already discovered objects of the Kuiper Belt to obtain more accurate orbits. Observations of the asteroids approaching the Earth (AAE) are of no less interest. These observations are made in the directions which are close to directions to the Earth and the Moon and are easier technically because the brightness of the AAE is inconsiderable.

Independent of the celestial mechanics program the determination of orbits of moving minor objects of the solar system, i.e. asteroids, comets, planetary satellites, etc., is possible by using various methods, including the apparent motion parameters method (so-called the PVD-method), which has been developed at the Pulkovo Observatory and tested on conventional ground-based observations (Kiselyov \& Bykov, 1976).

\section{The observations of the microlensing events}

The idea of simultaneous observations of microlensing events from the Earth's surface and from aboard a distant spacecraft, very remote from the Earth, was proposed for the first time by Gould in 1992 (Gould, 1992, 1994a, 1994b). This experiment with the system of three instruments placed into the points $L_{4}, L_{5}$, $T$ (Fig. 7, b)) could also provide unique information on a lens.

Observations of microlensing events, (Fig. 7, a)), using the on-board equipment are possible, however, only after the detection of these events by the ground-based observer teams (Gurevich et al. 1997; Narayan \& Bartelman 1996; Zylberajch 1995). After ground-based detection of the events the coordinates of suspected objects are introduced into the on-board observation program of each spacecraft. If it would be possible to observe the moments of transit of a brightness maximum of the lensed object (Fig. 7, b)) at the three vertices of the triangle $L_{4} T L_{5}$ it would allow us to estimate by the direct geometrical way the tangential component of the relative spatial path of a gravitational lens $(L)$ or of the lensed object $(S)$ image (the arrow $A$ ), the conventional linear tangential velocity $V_{\tau}$ being determined directly. The relative proper motion and its positional angle could be estimated for $L$ (Chubey 1998) if the conventional parallax or the direct distance $D_{O L}$ is known. As an alternative to the gravitational refraction which is of the order of two microarcseconds (Hosokawa et al., 1995), the photometric observations of the microlensing effect on the changes of 


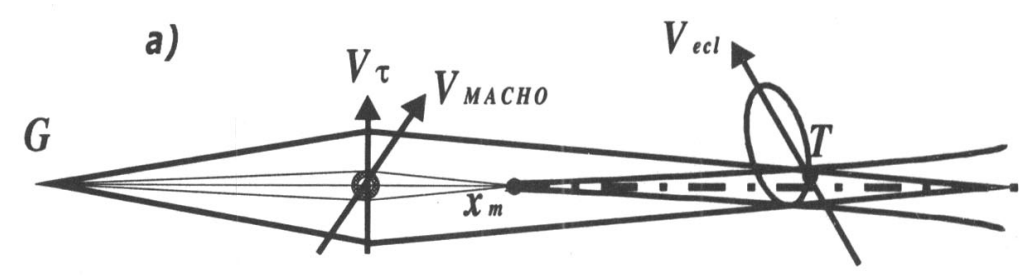

b)

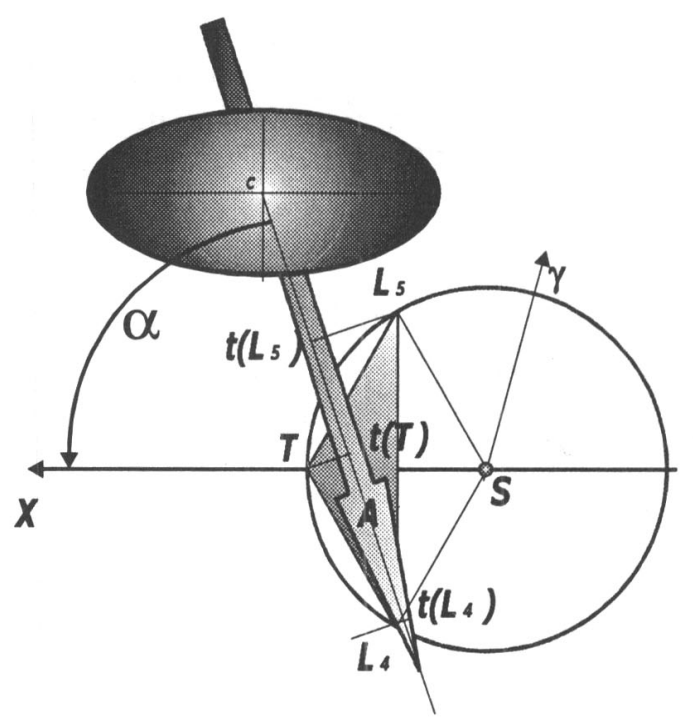

c)

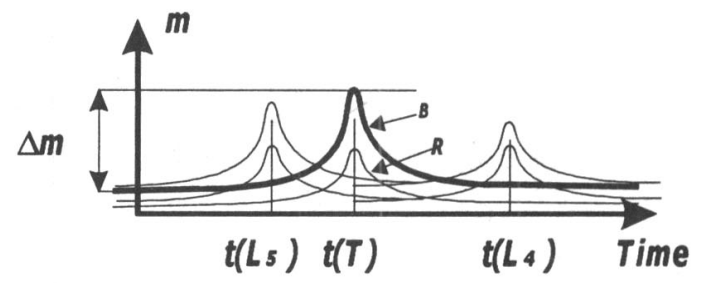

Figure 7. Observations of the microlensing events. a) The principal scheme. b) $A$, the path of a zone of the focused image brightness center $C$ through instruments in the points $L_{4}, T, L_{5}$. The brightness maxima will be observed at different moments. c) The brightness increment of $\Delta m$ should be observed through several filters (e.g., $B$ and $R$ ) with each instrument. 

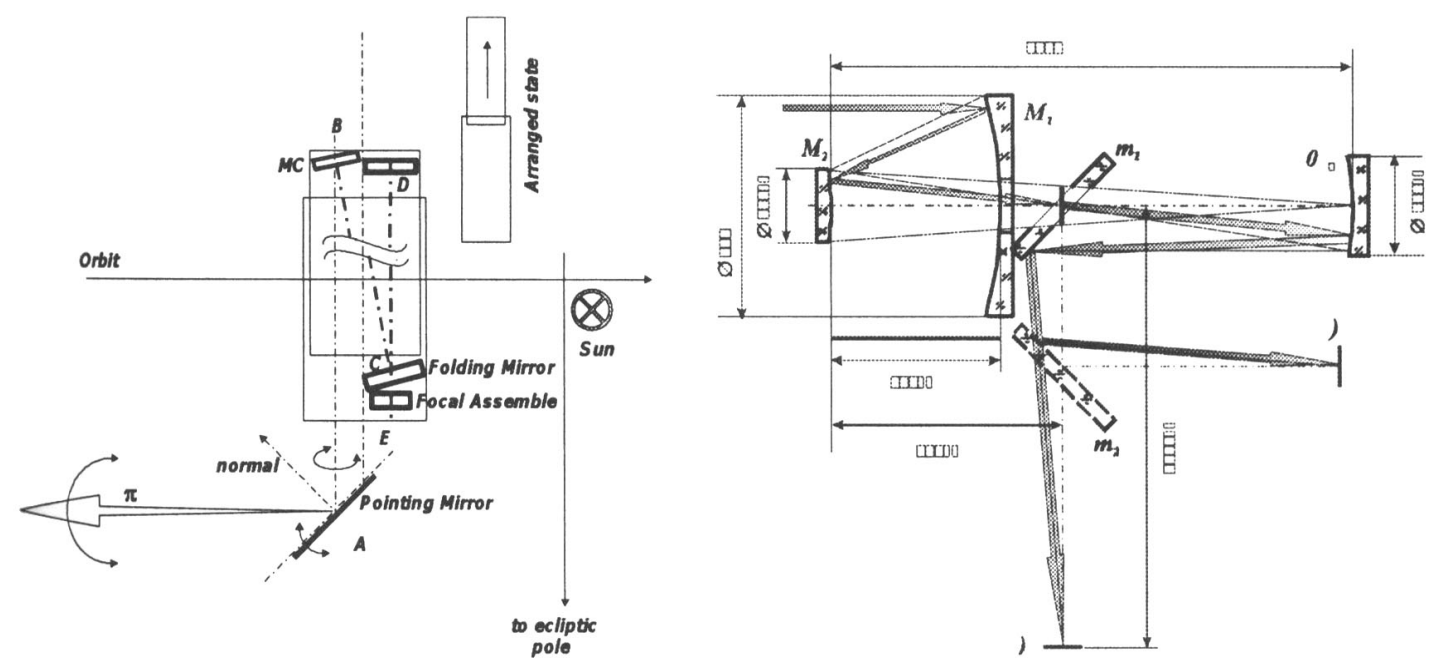

Figure 8. Left: the concept of the Schmidt space camera. Right: the instrument version of the Korsch three-mirror-system (Korsch, 1977) as designed by Tsukanova and Starichenkova (1997).

the observed light curves (Fig. 7, c)), being corrected for orbital movement, are more practicable.

\section{The instruments}

It is proposed to use an astrograph with limiting magnitudes of $21^{m}-22^{m}$ and with diffraction quality of images in the field of view with angular diameter $2 W=1^{\circ}$ as a basic instrument. Two optical schemes are being studied: the Schmidt space camera and the three-mirror system telescope of Korsch (1977). Both instruments should be equipped with the pointing mirror (Fig. 8) with an aperture of $500 \mathrm{~mm}$ and a focal length exceeding $5000 \mathrm{~mm}$, with a folded mounting, the CCD-mosaic as a light detector ( 4 lines by 5 modules $\approx 800 \times 800$ of $16 \mu$ pixels, scale $<40^{\prime \prime} / \mathrm{mm}$ or $\approx 0.66^{\prime \prime} /$ pixel, field of view $\left.\approx 35^{\prime} \times 45^{\prime}\right)$, the signal to noise ratio being $\geq 5$.

The astrometric star position determination accuracy is planned to be better than 10 mas, the photometric one being not worse than $0.02^{m}$ or $0.03^{m}$ for an individual measurement of objects of $17^{\mathrm{m}}$ to $19^{\mathrm{m}}$. The following characteristics and processes are permanently calibrated by the flexible check program aboard: the dark current, the non-uniformity of the sensitivity of the detector or "the flat field," and the record of damaged pixels. For the photometry systems the broadband $U B V R$ and integral ones are to be used.

The information acquisition is planned to be performed in the optimal frame addition mode, 96 frames per day. It would be possible to compress each frame up to density $p \approx 2$ bits per pixel (White \& Percival, 1994) or up to $4 \mathrm{MB}$ per frame (after on-board processing). The working brightness range is $\Delta V \in$ 
$\left(12^{m}-22^{m}\right)$ at the mission beginning. The daily astronomical information volume rate is 3 Gbytes. Addition of the solar physics information amounting to 6.5 Gbytes will result in $9.5 \mathrm{~GB}$ total information to be transferred each day requiring the capacity of the communication channel to be $\approx 110 \mathrm{~KB}$ per sec. To meet these requirements the design concept includes the folding phased antenna with the planned surface area of $\approx 25 \mathrm{~m}^{2}$.

We may conclude that there are evident scientific objectives in the creation of the ISSO. The idea to create a space radio interferometer on the same basis as that of the ISSO has been suggested in Kardashev et al. (1973). The gravitational features of these remarkable projects will probably be used in future space experiments.

\section{References}

Bakut P.A., Schulz, S.V., 1998, Kosmicheskiye Issledovaniya (Space Researches), 36, 407-416. (In Russian).

Chubey, M.S., 1998. Izvestiya GAO RAN (Pulkovo Observatory Reports). No. 213. 273-278 (In Russian).

Chubey, M.S., Kopylov, I.M., I'in, A.E., Gorshanov, D.L., Butkevich, A.G., Gritsuk, A.N., Savastenya, A.V., 1998, Trudy IV S'ezda Astronomicheskogo Obshchestva (Proceedings of the IVth Congress of the Astronomical Society). Moscow, GAISh, 103-109. (In Russian).

Chuchkov E.A. (Ed.), 1989, Cosmic ray intensity in interplanetary space. Observational data. Moscow.

Gould, A., 1992, Astrophys. J., 392, 442.

Gould, A., 1994a, Astrophys. J., 421, L71.

Gould, A., 1994b, Astrophys. J., 421, L75.

Grigoryev, V.M., 1993, Spaceborne solar stereoscope experiment in Solar Physics, Solar Phys., 148, 386-391.

Gurevich, A.V., Zybin, K.P. Sirota, V.A., 1997. Uspekhi Fizicheskikh Nauk (Progress in the Physical Sciences), 167, 913-943.

Hosokawa, M., Ohnishi, K., Fukushima, T. and Takeuti, M., 1995, "Astronomical and Astrophysical Objectives of Sub-Milliarcsecond Optical Astrometry," E. Høg and P.K Seidelmann (Eds.). IAU. Printed in The Netherlands. 305-308.

Kardashev, N.S., Parijski, Yu.N., Umarbayeva, N.D., 1973, Izvestiya SAO (Reports of the Special Astrophysical Observatory), 5, 16-29. (In Russian).

Kiselyov, A.A., Bykov, O.P., 1976, Russ. A.J., 53, 879-888.

Korsch, D., 1977, Anastigmatic three-mirror telescope. Appl. Opt., No. 8, 2074-2077.

Narayan, R. and Bartelmann, M., 1996, Lectures on Gravitational Lensing. Max-Planck-Inst. MPA 961, June 1996.

Paczynski, B., 1986, Astrophys. J., 304, 1.

Standish Jr., E.M., 1990, Astron. Astrophys., 233, 252-271. 
Tsukanova G.I., Starichenkova, V.D., 1997, Journ. of Optical Technology, 64, 64-67.

White, R.L. and Percival, J.W., 1994, SPIE, 2199, 703-713

Zabelina, I.A., 1997, Optical Journal (Opticheskij Jurnal), No. 7, p. 3. (In Russian).

Zylberajch, S., 1995, In: Conference Proceedings Vol. 47, 'Frontier Objects in Astrophysics and Particle Physics," F. Giovannelli and G. Mannocchi (Eds.), SIF, Bologna, 105-120. 